BULL. AUSTRAL. MATH. SOC.

$47 G 05,46 B 99$

VOL. $19(1978), 147-149$.

\title{
Some new function spaces and their tensor products
}

\author{
Mario M. Milman
}

The theory of the mapping properties of an integral operator $K: A \rightarrow B$, where $K$ is a fixed operator and the spaces $A, B$ are to be found, has come to be called the theory of admissibility. In particular the behaviour of solutions to integral equations can be simply described within the framework of admissibility theory. In this theory a central role is played by Banach function spaces as defined in [4]; the wider the variety of Banach function spaces with which we are equipped, the more precisely can we specify the asymptotic or local properties of the solutions to an integral equation.

This thesis studies admissibility problems, which by duality are equivalent to the embedding of the projective tensor product to two Banach function spaces into a third Banach function space. A fundamental role in its development is played by a construction of Banach function spaces from others originally due to Calderón [1]; using this construction the thesis extends, unifies, and considerably simplifies previous work of $0^{\prime} \mathrm{Neil}$ [5] and Corduneanu [3].

In Chapter 1 studies are made of the basic construction of spaces $A^{-1}(X)$ first used by Calderon [1]. Let $X(\Omega)$ be a Banach function space on a (nonatomic, $\sigma$-finite, complete) measure space $\Omega, \Sigma(X)$ its unit ball, and $A$ a Young's function. For each pair $(X(\Omega), A)$ define (1.0) $A^{-1}(X)(\Omega)=\{f \in M(\Omega) \mid \exists \lambda>0$ and

$$
g \in \Sigma(X) \rightarrow|f(x)| \leq \lambda A^{-1}(|g(x)|) \mid .
$$

Received 27 September 1978. Thesis submitted to the Australian iiational University, May 1978. Degree approved, August 1978. Supervisor: Dr Thomas K. Donaldson. The Editor thanks Dr Donaldson for writing this abstract. 
The construction (1.0) produces a wide variety of spaces including many familiar ones. For example, $A^{-1}\left(L^{1}\right)=L_{A}$, the classical Orlicz spaces. Many subsequent proofs and definitions depend on the properties of this construction.

In Chapter 2 the class of OCLHZ spaces $\Lambda\left(A, \phi_{X}, \phi_{Y}\right)$ and $\Lambda\left(A, \phi_{X}, \phi_{Y}\right)^{\sim}$ is introduced, which provides the main subject of this thesis. Let $A$ be a Young's function, $X, Y$ rearrangement invariant spaces, and $\phi_{X}, \phi_{Y}$ their fundamental functions (as defined in [6]). Let $f^{*}$ be the nonincreasing rearrangement of $f \in M(\Omega)$ onto $(0, \infty)$ and

$$
f^{* *}(x)=\frac{1}{x} \int_{0}^{x} f^{*}(t) d t
$$

and finally let $d \mu(t)=\phi_{Y}(t) \frac{d t}{t}$. The norms of $\Lambda\left(A, \phi_{X}, \phi_{Y}\right)$ and $\Lambda\left(A, \phi_{X}, \phi_{Y}\right)^{\sim}$ are then defined

$$
\begin{aligned}
& \text { (1.l) }\|f\|_{\Lambda\left(A, \phi_{X}, \phi_{Y}\right)}=\inf \left\{\alpha>0 \mid \int_{0}^{\infty} A\left[f^{* *}(t) \phi_{X}(t) \alpha^{-1}\right\} d \mu(t) \leq 1\right\} \text {, }
\end{aligned}
$$

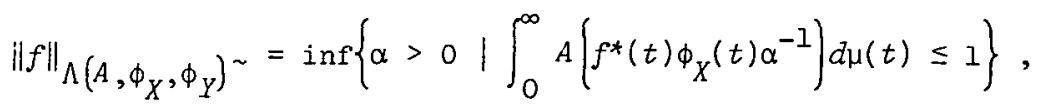

and the corresponding spaces consist of all measurable functions for which the norms (1.1) are finite. For particular specializations of the spaces $X, Y$ and the Young's function $A$, we get

$$
\begin{gathered}
\Lambda(A, 1, t)^{\sim}=L_{A} \text { for } y=L^{1}, X=L^{\infty}, \\
\Lambda\left(t^{q} \cdot q^{-1}, t^{(1 / p)}, 1\right)=L(p, q) \text { for } y=L^{\infty}, X=L^{p}, A(t)=(1 / q) t^{q} .
\end{gathered}
$$

Theorems are then proved relating $\Lambda\left(A, \phi_{X}, \phi_{Y}\right)$ and $\Lambda\left(A, \phi_{X}, \phi_{Y}\right)^{\sim}$, giving conditions for separability, or reflexivity and calculating the associate spaces and dual spaces of OCLHZ spaces. For instance, using the methods described, explicit conditions are stated under which

$$
\Lambda\left(A, \phi_{X}, \phi_{Y}\right)^{\prime} \cong \Lambda\left(\bar{A}, \psi, \phi_{Y}\right) \text { where } \psi(t)=\phi_{X^{\prime}}(t) / \phi_{Y}(t)
$$

and similarly conditions for reflexivity. 
These spaces blend the characteristic features of the Lorentz spaces $L(p, q)$ and the Orlicz spaces, giving a much more general "scale" which includes both types. They allow a very much finer study of stability behaviour for integral operators, considerably extend the available range of "concrete" function spaces, and provide a setting for much more general and illuminating versions of results known for Orlicz spaces or $L(p, q)$ spaces.

In Chapter 3 the classical theory of weak interpolation [2] is extended to OCLHz spaces, providing interpolation theorems which hold simultaneously for Orlicz spaces and $L(p, q)$ spaces. Chapters 4,5 study embeddings of tensor products of OCHLZ spaces, extending and complementing work of $\mathrm{O}^{\prime} \mathrm{Neil}$. Among the achievements of this theory is a drastic simplification of the lengthy proofs of [5]. Similar theorems are also shown for convolution products. Finally Chapter 6 gives applications of this work to admissibility of integral operators along the lines of previous work by Corduneanu [3].

\section{References}

[1] A.P. Calderón, "Intermediate spaces and interpolation, the complex method", Studia Math. 24 (1964), 113-190.

[2] A.P. Calderón, "Spaces between $L^{I}$ and $L^{\infty}$ and the theorem of Marcinkiewicz", Studia "lath. 26 (1966), 273-299.

[3] Constantin Corduneanu, Integral equations and stability of feedback systems (Mathematics in Science and Engineering, 104. Academic Press, New York and London, 1973).

[4] W.A.J. Luxemburg, "Banach function spaces" (Thesis, Delft Institute of Technology, Assen, 1955). [Quoted from W.A.J. Luxemburg and A.C. Zaanen, Riesz spaces, Volume I (Horth-Holland, Amsterdam, London, 1971), p. 509.]

[5] Richard O'Neil, "Integral transforms and tensor products of Orlicz spaces and $L(p, q)$ spaces", $J$. Analyse Math. 21 (1968), 1-276.

[6] M. Zippin, "Interpolation of operators of weak type between rearrangement invariant function spaces", J. Functional Analysis 7 (1971), 267-284. 